\title{
AFP and CA-125 as an accurate risk factor to predict eye metastasis in hypertension patients with liver Carcinoma囚 A STROBE-compliant article
}

\section{Jing Tang}

The First Affiliated Hospital of Nanchang University

\section{Li-Juan Zhang}

The First Affiliated Hospital of Nanchang University

\section{Yan-Cheng Huang}

The First Affiliated Hospital of Nanchang University

\section{Rong Huang}

The First Affiliated Hospital of Nanchang University

\section{Hui-Ye Shu}

The First Affiliated Hospital of Nanchang University

Jie-Li Wu

Institute of Xiamen University, Medical College of Xiamen University

Qiu-Yu Li

The First Affiliated Hospital of Nanchang University

\section{Ting Su}

Harvard Medical School

\section{Yi-Cong Pan}

The First Affiliated Hospital of Nanchang University

\section{Rong-Bin Liang}

The First Affiliated Hospital of Nanchang University

Yi Shao ( $\nabla$ freebee99@163.com )

The First Affiliated Hospital of Nanchang University

\section{Research Article}

Keywords: AFP, CA-125, ocular metastasis, primary liver cancer, hypertension, history of hepatitis B Posted Date: August 10th, 2021

DOl: https://doi.org/10.21203/rs.3.rs-754934/v1 
License: (c) (i) This work is licensed under a Creative Commons Attribution 4.0 International License. Read Full License 


\section{Abstract \\ Purpose}

Primary liver carcinoma is a common malignant tumor. In this study, we analyzed the differences between hypertension patients with ocular metastasis of liver cancer and those with metastases to other sites, the correlation between history of HBV and liver cancer metastasis, and independent risk factors for ocular metastasis.

\section{METHODS}

We used treatment records from 488 patients with metastases of primary liver cancer from August 2001 to May 2015, divided into two groups based on metastatic sites: OM (ocular metastasis) and NOM (nonocular, other sites of metastasis) groups. The Student's $t$ test and Chi-square test were used to assess the significance of differences between the groups and define the relationship between history of HBV and ocular metastasis of liver cancer. Binary logistic regression analysis was used to identify indicators of ocular metastasis of liver cancer and receiver operating curve (ROC) analyses to estimate their diagnostic value.

\section{RESULTS}

No significant differences in sex, age, tumor stage, pathological type, or treatment were identified between the OM and NOM groups, while the prevalence of HBV was higher in the former than that in latter, confirming the association between history of HBV and ocular metastasis. Binary logistic regression demonstrated that AFP and CA-125 were independent indicators of liver metastasis (both $\mathrm{P}<0.001$ ). ROC curve analyses generated cut-off values for AFP and CA-125 of $957.2 \mathrm{ng} / \mathrm{ml}$ and $114.25 \mathrm{U} / \mathrm{ml}$, respectively, with corresponding AUC values of 0.739 and 0.810 . The specificity of the combination of AFP and CA-125 was higher than either factor separately.

\section{Introduction}

Hypertension is a chronic disease with functional or organic damage of heart, brain, kidney and other organs, which brings a huge health burden to the society. Primary liver cancer (PLC) is a common disease worldwide, particularly in developing countries (1). Hepatocellular carcinoma (HCC) is the main pathological type of PLC, and exhibits high malignancy and strong invasiveness, resulting in poor prognosis and a heavy economic burden of treatment (2). Worldwide, patients with hepatitis $B$ virus (HBV) infection exceed 300 million, and many liver diseases, including liver cancer, can be secondary to HBV infection (3). Moreover, chronic hepatitis virus infection not only affects the liver, but also extrahepatic organs, leading to severe extrahepatic lesions such as dry eye, Mooren's ulcer, and retinopathy. HBV infection is clearly linked to dry eye syndrome (4). Extrahepatic metastasis is an 
indicator of prognosis in patients with PLC, and different metastatic sites are associated with distinct survival rates (5). A survey of 419 patients with HCC who had extrahepatic metastases found that the most common sites of extrahepatic metastases are lung, bone, lymph node, and adrenal gland in that order (6). The eye is a rare site of distant hepatic metastasis of PLC, and most patients with ocular metastases also have metastases at other sites. Hypertensive patients are prone to arteriosclerosis, which leads to liver blood supply insufficiency and liver function damage. Related clinical symptoms can cover up the signs of liver cancer, and the early clinical symptoms of HCC are not obvious; hence, patients can be completely unaware of their disease progression and even metastasis (7). Once a distant metastasis occurs, treatment is extremely difficult, and patient prognosis is poor. At present, the diagnosis of PLC mainly depends on imaging (ultrasound and magnetic resonance imaging (MRI) (8), clinicopathological correlation, and application of immunohistochemical markers (9); however, these methods are inefficient for early detection of metastases, hence a simple and effective clinical diagnostic indicator is needed to improve the early diagnosis rate of ocular metastasis from PLC.

\section{Materials And Methods}

\section{Study design}

This study met the requirements of the Declaration of Helsinki and was licensed by the Medical Ethics Committee of the First Affiliated Hospital of Nanchang University. The study design followed relevant regulations and guidelines. Hypertensive patients with PLC metastases $(n=488)$, admitted from August 2001 to May 2015, were enrolled in the study. The inclusion criteria are: 1) hypertension (systolic blood pressure $>140 \mathrm{mmHg}$ or diastolic blood pressure $>90 \mathrm{mmHg}$ ); 2 ) without contraindications in MRI, CT and other imaging examinations; 3) Canceration of liver tissue (Figure 1); 4) ocular metastasis of hepatocellular carcinoma (Figure 2). Diagnosis was determined by imaging (ultrasound, computed tomography (CT), MRI) and histopathological biopsy. A review of the medical records of the patients revealed that those with ocular metastases of liver cancer also had metastases at other sites. Therefore, patients were divided into two groups, according to the tumor metastasis sites: OM (PLC with ocular metastasis) and NOM group (PLC with non-ocular, but other metastasis sites) groups. Exclusion criteria for the OM group were: 1) primary malignant tumor of the eye; 2 ) benign tumor of the eye; 3 ) primary liver cancer patients with metastases in other sites (intrahepatic, portal system, lung, bone, etc.).

\section{Data collection}

Relevant clinical data were collected retrospectively from the medical records of each subject, including sex, age, history of HBV, clinical stage, pathological type, treatment methods, and other clinical features, serum markers (AFP, CEA, CA-125, CA-199, CA-153, CA-724), blood lipid indicators (lipoprotein a (Lp(a)), apolipoprotein $B(A p o B)$, apolipoprotein A1 (ApoA1), low-density lipoprotein (LDL), high-density lipoprotein ( $\mathrm{HDL}$ ), triglycerides (TG), total cholesterol (TC)), serum calcium concentration, hemoglobin concentration, ferritin, alkaline phosphatase (ALP), and other serum indicators. All clinical data were collected at initial diagnosis. 


\section{Statistical analysis}

Student's $t$ tests and Chi-square tests were used to evaluate the significance of differences in clinical characteristics between the OM and NOM groups. Binary logistic regression analysis was used to identify indicators of ocular metastasis of liver cancer. ROC curve analysis was conducted and the area under the curve (AUC) used to assess the accuracy of risk factors as diagnostic indicators. $P<0.05$ was considered statistically significant. SPSS 25.0 (SPSS, IBM, USA), MedCalc 19.0.5 (MedCalc Ostend, Belgium), and Excel 2019 (Microsoft Corp, Redmond, WA, USA) software were used for statistical analyses.

\section{Results}

\section{Demographic and clinical characteristics}

In this study we reviewed data from 488 patients, including 21 in the OM and 467 in the NOM groups. Clinical characteristics, including sex, clinical stage, pathological type, and treatment methods did not differ significantly between the OM and NOM groups (Student's $t$-test and Chi-square test). The OM group was significantly older $(57.7 \pm 12.2$ years $)$ than the NOM group $(51.5 \pm 13.8$ years $)(P<0.05)$. There was also a significant difference in history of HBV between the two groups $(P<0.05)$, confirming that ocular metastasis of PLC is associated with history of HBV. The clinical details of all subjects are presented in Table 1.

\section{Differences in clinical characteristics and risk factors for $\mathrm{OM}$}

CEA, CA-199, CA-153, CA-724, TC, TG, HDL, LDL, ApoA1, ApoB, Lp(a), calcium, Hb, ferritin, ALP, and other serological indicators did not differ significantly between the OM and NOM groups, while AFP and CA-125 were significantly higher in the OM group $(P<0.001)$ (Table 2). Binary logistic regression analysis determined that AFP and CA-125 were associated with liver cancer metastasis (Table 3).

\section{Cut-off, sensitivity, specificity, and AUC values of AFP and CA-125 for diagnosing ocular metastases of PLC}

Figure 3 shows the ROC curves for AFP and CA-125, as independent risk factors for ocular metastasis of liver cancer, and their AUC, specificity, sensitivity, and cut-off values. The cut-off values for AFP and CA125 were 957.2 and $114.25 \mathrm{U} / \mathrm{ml}$ and AUC values were 0.739 and 0.810 , respectively. AFP had a higher sensitivity value than CA-125, while the specificity of CA-125 was higher than that of AFP. Figure 4 shows a comparison of the ROC curves for AFP, CA-125, and the combination of AFP and CA-125 (AFP+CA-125). The AUC, sensitivity, specificity, and cut-off values for AFP, CA-125, and AFP+CA-125 are presented in Table 4. Among them, AFP+CA-125 had the largest AUC (0.875), AFP the highest sensitivity (95.2\%), and AFP+CA-125 the highest specificity (88.4\%); all results were statistically significant.

\section{Discussion}


Liver carcinoma ranks second among the causes of cancer mortality globally (10). PLC pathological types include: intrahepatic cholangiocarcinoma (iCCA), HCC, and other rare types (11). Among these, HCC is the primary pathological type, accounting for approximately $80 \%$ of the total (12). Wang et al. confirmed this through statistical analysis of 2172 patients with histologically confirmed PLC, among which were $1,823 \mathrm{HCC}$ and 238 iCCA patients, accounting for $83.9 \%$ and $11.0 \%$, respectively (13). Therefore, the PLC analyzed in this article was primarily HCC.

The incidence of $\mathrm{HCC}$ is increasing, and it is associated with a high mortality rate. Cirrhosis caused by HBV and hepatitis $\mathrm{C}$ is a significant risk factor for HCC globally (14); however, in most areas of China, HBV is the most important pathogen in the context of PLC. (13,15-16). The pathogenesis of HBV is clear. The integrated viral sequence produces different mutations in the preS/S gene, due to replication defects, which can induce various tumorigenesis mechanisms, thereby contributing to HCC (17).

Infection with HBV can cause a variety of liver lesions and illness; for example, fulminant cirrhosis, chronic hepatitis, hepatic failure, acute hepatitis, and HCC (3). Cirrhosis is an intermediate process, through which viral hepatitis develops into liver cancer. Most HCC patients with a history of viral hepatitis have experienced cirrhosis (18). Mahmood et al. (19) conducted a study on cirrhosis and found that, among the 137 patients with cirrhosis who participated in the trial, the causes of cirrhosis included: hepatitis C virus (61\%), HBV (32\%), alcoholism (3\%), and primary biliary cirrhosis (3\%). In addition, $47 \%$ of participants had ocular complications. Giovannini et al. (20) reported that $78 \%$ of patients with primary biliary cirrhosis suffered from dry eye. It is clear that HBV contributes to liver cirrhosis and even liver cancer, and the former can cause ocular complications; therefore, there is evidence for an indirect relationship between history of HBV and ocular lesions. The mechanism underlying this relationship is currently unclear; however, experiments in mice confirmed that SMAD3 mediates the majority of profibrotic activity, since liver cirrhosis, proliferative vitreoretinopathy, and ocular capsule injury are alleviated in SMAD3-null mice (21).

HBV can also cause eye lesions directly. A 72-year-old chronic HBV patient was also diagnosed with orbital MALT lymphoma, with a clinical manifestation of bilateral ocular protrusion and slight limitation of eye movement, providing evidence for a possible association between HBV and ocular lesions (22). There is a risk of eye complications during the prevention and treatment of HBV. HBV vaccine, either alone or administered with other vaccines, appears to be the leading offender in causing uveitis (23). Kwon et al. (24) reported a 15-year-old boy who developed glaucoma while using interferon alpha therapy for chronic HBV. The glaucoma symptoms were improved after discontinuation of interferon therapy.

Liver cancer is frequently occult; $70 \%$ of patients with PLC have detectable metastases at initial diagnosis, while metastases account for $90 \%$ of the total cancer-related mortality rate (25). Different distant extrahepatic metastases vary greatly in terms of mortality rates. The lung is common metastatic site of PLC, while brain metastasis is rare, and patients with brain metastasis have the worst prognosis (5). Other rarer cases of HCC metastatic sites include thyroid (26) and nodal (27). 
As there is no lymphatic system in the eye, it is rarely a site of malignant tumor metastasis; however, malignant tumors can still be transferred to the eye via the blood system. Therefore, blood-rich areas, such as the posterior choroid of the uveal structure, are prone to ocular metastases. The appearance of ocular metastases is also conclusive evidence of tumor metastasis (28).

Most patients with stage 4 cancer (distal tumor metastasis), including distant ocular metastases, have clear primary sites (29). The most common among them for ocular metastases are breast and lung (28). Primary cancer sites also include: pancreas (1\%), thyroid (1\%), prostate (2\%), lung carcinoid (2\%), cutaneous melanoma (2\%), gastrointestinal (GI) tract (4\%), kidney (4\%), lung (26\%), breast (37\%), other sites (3\%), and unknown (16\%) (30). All of the patients in our study with eye metastases also had other sites of metastasis. The eye is the terminal organ of liver cancer metastasis. Once metastasis occurs, treatment is difficult and the prognosis poor. Therefore, we compared patients with ocular metastasis to those with other metastasis sites, to identify indicators of ocular metastasis of liver cancer and improve the early diagnosis rate. We found that the OM group was older than the NOM group $(P<0.05)$, likely because elderly patients have a higher prevalence of diabetes, poorer physical fitness, and cannot tolerate higher-intensity treatment (31). In addition, due to the association between HBV and ocular lesions, patients with a history of HBV had higher rate of metastases to the eye than to other sites. During clinical diagnosis of PLC, whether the patient has a history of HBV should be determined, to inform subsequent treatment.

Lp(a), ApoB, ApoA1, LDL, HDL, TG, TC, hemoglobin, ferritin, and ALP are commonly used as indicators for evaluating blood lipids and liver function. Statistical analysis did not identify any significant differences between the above indicators in the OM and NOM groups. The serum biomarkers, CA-153, CA-199, CA125, CEA, AFP, and CA-724 are tumor markers and have clear predictive significance for a variety of tumors (32-35). In patients with HBV and hepatitis $C$ virus, particularly combined evaluation of AFP, CEA, CA-125, CA-153, and CA-199 have already been implemented (36). Among them, multiple roles for AFP and CA-125 have been confirmed. AFP is a plasma protein produced by embryonic tissue. Healthy adult adults have very low levels of AFP, and some primary cancers can cause significant increases in this factor; therefore, it can be used to screen for tumors and other pathologies in adults (37), including primary HCC (38), and gastric cancer (39). CA-125 levels are associated with lymphangioleiomyomatosis (LAM), and elevated CA-125 may indicate LAM with pleural effusion, leading to decreased lung function (40). Karimi-Zarchi et al. (41) reported that CA-125 can be used for clinical prediction of endometriosis, while another report showed CA-125 as useful for surveillance in ovarian cancer (42).

Non-invasive standard imaging methods, such as MRI, dynamic multi-phase multi-row computed tomography, and ultrasound, are used to diagnose HCC (43). Serum biomarker assays are reproducible, simple, and rapid, relative to traditional diagnostic methods. Our statistical analyses confirmed the diagnostic value of AFP and CA-125 for liver metastasis.

Finally, we determined the value of AFP, CA-125, and AFP+CA-125 as diagnostic indicators for ocular metastasis of liver cancer by binary logistic regression analysis and plotting ROC curves. The results 
indicate that AFP has the highest sensitivity and can be used for early screening of ocular metastases from liver cancer. If AFP rises above $957.2 \mathrm{U} / \mathrm{ml}$, the patient has a higher probability of terminal ocular metastasis. AFP+CA-125 has the highest specificity, and is of great significance for the diagnosis of ocular metastasis from liver cancer.

Our research has certain limitations. First, the sample size of this retrospective study was small, particularly for the OM group. Second, because all patients in the OM group had other sites of metastases simultaneously, confounding factors were inevitable. Furthermore, there were missing items in various clinical statistical analyses, which will have reduced the accuracy of the results. Finally, all subjects were diagnosed and treated in the same hospital, and it is difficult to exclude selection bias; therefore, the accuracy of the conclusions from the results of this study require confirmation in investigations with large samples and multiple centers.

\section{Declarations}

Consent for publication $₫$ All authors agree to publish the manuscript.

Availability of data and material: The datasets used and analysed during the current study are available from the corresponding author on reasonable request.

Competing interests: This was not an industry supported study. The authors report no conflicts of interest in this work.

Funding: The Central Government Guides Local Science and Technology Development Foundation(No: 20211ZDG02003); Key Research Foundation of Jiangxi Province (No: 20181BBG70004, 20203BBG73059); Excellent Talents Development Project of jiangxi Province『No: 20192BCBL23020®; Natural Science Foundation of jiangxi Province『No: 20181BAB205034『; Grassroots Health Appropriate Technology "Spark Promotion Plan" Project of Jiangxi Province(No:20188003); Health Development Planning Commission Science Foundation of Jiangxi Province (No: 20201032,202130210); Health Development Planning Commission Science TCM Foundation of Jiangxi Province (No: 2018A060,2020A0087); Education Department Foundation of Jiangxi Province『No: GJJ200157, GJJ200159, GJJ200169囚

Author contributions: Each author has made a contribution. Jing Tang is responsible for the writing of the manuscript, Li-Juan Zhang and Yan-Cheng Huang are responsible for the formulation and evaluation of the experimental scheme,Rong Huang and Hui-Ye Shu are responsible for recruiting and screening experimental subjects, Jie-Li Wu and Qiu-Yu Li are responsible for preparing experimental materials and executing the experiment, Ting Su囚Yi-Cong Pan and Qiu-Yu Li are responsible for data analysis and collation. Shao $\mathrm{Yi}$ is responsible for the revision of the paper.

Ethical Statement: All research methods were approved by the committee of the medical ethics of the First Affiliated Hospital of Nanchang University and were in accordance with the 1964 Helsinki 
declaration and its later amendments or comparable ethical standards. All subjects were explained the purpose, method, potential risks and signed an informed consent form.

\section{Conclusion}

In summary, we found that patients who had PLC metastasis with a history of HBV were more likely to have ocular metastases than those without. Increases in the serum biomarkers, AFP and CA-125, are associated with an increased likelihood of ocular metastasis. AFP, CA-125, and AFP combined with CA125 can be used as diagnostic indicators for ocular metastasis in patients with PLC with metastasis.

\section{References}

1 Zhang, Y., et al. (2015). "International trends in primary liver cancer incidence from 1973 to 2007." BMC Cancer 15: 94.

2 Wang, S., et al. (2016). "Improved survival of patients with hepatocellular carcinoma and disparities by age, race, and socioeconomic status by decade, 1983-2012." Oncotarget 7(37): 59820-59833.

3 Liang, T. J. (2009). "Hepatitis B: the virus and disease." Hepatology 49(5 Suppl): S13-21.

4 Tsoumani, A., et al. (2013). "Treatment and non-treatment related ocular manifestations in patients with chronic hepatitis B or C." Eur Rev Med Pharmacol Sci 17(8): 1123-1131.

$5 \mathrm{Wu}$ W, He X, Andayani D, et al. Pattern of distant extrahepatic metastases in primary

liver cancer: a SEER based study. Journal of Cancer. 2017;8(12):2312-2318.

6 Aino, H., et al. (2014). "Clinical characteristics and prognostic factors for advanced hepatocellular carcinoma with extrahepatic metastasis." Mol Clin Oncol 2(3): 393-398.

7 McMahon, B., et al. (2016). "Hepatitis-Associated Liver Cancer: Gaps and Opportunities to Improve Care." J Natl Cancer Inst 108(4).

8 Cassinotto, C., et al. (2017). "Diagnosis of hepatocellular carcinoma: An update on international guidelines." Diagn Interv Imaging 98(5): 379-391.

9 Vyas, M. and D. Jain (2018). "A practical diagnostic approach to hepatic masses." Indian J Pathol Microbiol 61(1): 2-17.

10 McGlynn, K. A., et al. (2015). "Global epidemiology of hepatocellular carcinoma: an emphasis on demographic and regional variability." Clin Liver Dis 19(2): 223-238.

11 Sia, D., et al. (2017). "Liver Cancer Cell of Origin, Molecular Class, and Effects on Patient Prognosis." Gastroenterology 152(4): 745-761. 
12 Madkhali, A. A., et al. (2015). "Surgical treatment for hepatocellular carcinoma."

Saudi J Gastroenterol 21(1): 11-17.

13 Wang, M., et al. (2017). "Contribution of hepatitis B virus and hepatitis C virus to liver cancer in China north areas: Experience of the Chinese National Cancer Center." Int $J$ Infect Dis 65: 15-21.

14 Mittal, S. and H. B. El-Serag (2013). "Epidemiology of hepatocellular carcinoma: consider the population." J Clin Gastroenterol 47 Suppl: S2-6.

$15 \mathrm{Li}, \mathrm{H}$. M., et al. (2015). "Hepatitis B virus genotypes and genome characteristics in China." World J Gastroentero/ 21(21): 6684-6697.

16 Ye, Y. F., et al. (2015). "Hepatitis B virus infection and risk of nasopharyngeal

carcinoma in southern China." Cancer Epidemiol Biomarkers Prev 24(11): 1766-1773.

17 Li, Y. W., et al. (2016). "Hepatocellular carcinoma and hepatitis B surface protein." World J Gastroenterol 22(6): 1943-1952.

18 Ringelhan, M., et al. (2017). "Viral hepatitis and liver cancer." Philos Trans R Soc Lond B Biol Sci372(1732).

19 Mahmood, K., et al. (2008). "Serum retinol binding protein as an indicator of vitamin A status in cirrhotic patients with night blindness." Saudi J Gastroentero/ 14(1): 7-11.

20 Giovannini, A., et al. (1985). "Patterns of lacrimal dysfunction in primary biliary cirrhosis." $\mathrm{Br} J$ Ophthalmol69(11): 832-835.

21 Flanders, K. C. (2004). "Smad3 as a mediator of the fibrotic response." Int J Exp Patho/ 85(2): 47-64.

22 Lin, P. H., et al. (2017). "Bilateral orbital marginal zone B-cell lymphoma of the mucosa-associated lymphoid tissue in a patient with hepatitis B virus infection." Am J Ophthalmol Case Rep 7: 27-30.

23 Benage, M. and F. W. Fraunfelder (2016). "Vaccine-Associated Uveitis." Mo Med 113(1): 48-52.

24 Kwon, Y. S., et al. (2001). "Development of glaucoma in the course of interferon alpha therapy for chronic hepatitis B." Yonsei Med J 42(1): 134-136.

25 Arvelo, F., et al. (2016). "Cancer and the metastatic substrate." Ecancermedicalscience 10: 701.

26 Liang, H. H., et al. (2007). "Thyroid metastasis in a patient with hepatocellular carcinoma: case report and review of literature." World J Surg Oncol 5: 144.

27 Liu, Y. J., et al. (2017). "Composite hepatocellular carcinoma and small cell carcinoma with early nodal metastasis: A case report." Medicine (Baltimore) 96(34): e7868. 
28 Konstantinidis, L. and B. Damato (2017). "Intraocular Metastases-A Review." Asia Pac J Ophthalmol (Phila) 6(2): 208-214.

29 Cohen, V. M. (2013). "Ocular metastases." Eye (Lond) 27(2): 137-141.

30 Shields, C. L., et al. (2018). "Uveal Metastasis: Clinical Features and Survival Outcome of 2214 Tumors in 1111 Patients Based on Primary Tumor Origin." Middle East Afr J Ophthalmol 25(2): 81-90.

31 Guo, H., et al. (2017). "Hepatocellular carcinoma in elderly: Clinical characteristics, treatments and outcomes compared with younger adults." PLoS One 12(9): e0184160.

$32 \mathrm{Kim}, \mathrm{N}$. H., et al. (2017). "Serum CEA and CA 19-9 Levels are Associated with the Presence and Severity of Colorectal Neoplasia." Yonsei Med J 58(5): 918-924.

33 Zhao, S., et al. (2015). "Levels of CEA, CA153, CA199, CA724 and AFP in nipple discharge of breast cancer patients." Int J Clin Exp Med 8(11): 20837-20844.

34 Zou, D., et al. (2006). "[Expression and significance of CEA and CA153 in pleural fluid of patients with lung cancer]." Zhongguo Fei Ai Za Zhị 9(4): 337-339.

35 Hogendorf, P., et al. (2017). "A Panel of CA19-9, Ca125, and Ca15-3 as the

Enhanced Test for the Differential Diagnosis of the Pancreatic Lesion." Dis Markers 2017: 8629712.

36 Assmar, M., et al. (2016). "Combined Evaluation of AFP, CA15-3, CA125, CA19-9, and CEA Tumor Markers in Patients with Hepatitis B and C." Iran J Public Health 45(12): 1645-1651.

37 Adigun, O. O. and S. Khetarpal (2019). Alpha Fetoprotein (AFP, Maternal Serum Alpha Fetoprotein, MSAFP). StatPearls. Treasure Island (FL), StatPearls Publishing LLC.

38 Ahmed Mohammed, H. F. and L. R. Roberts (2017). "Should AFP (or any biomarkers) be used for HCC surveillance?" Curr Hepatol Rep 16(2): 137-145.

39 Sun, W., et al. (2017). "Novel characteristics of alpha-fetoprotein (AFP)-producing gastric cancer." Oncotarget 8(60): 101944-101951.

40 Glasgow, C. G., et al. (2018). "CA-125 in Disease Progression and Treat ment of Lymphangioleiomyomatosis." Chest 153(2): 339-348.

41 Karimi-Zarchi, M., et al. (2016). "Correlation of CA-125 serum level and clinico-pathological characteristic of patients with endometriosis." Int J Reprod Biomed (Yazd) 14(11): 713-718. 
42 Esselen, K. M., et al. (2016). "Use of CA-125 Tests and Computed Tomographic Scans for Surveillance in Ovarian Cancer." JAMA Oncol 2(11): 1427-1433.

43 Wang, C. H., et al. (2015). "Current trends and recent advances in diagnosis,

therapy, and prevention of hepatocellular carcinoma." Asian Pac J Cancer Prev

\section{Tables}

Table 1 The clinical characteristics of patients with metastases of primary liver cancer 


\begin{tabular}{|c|c|c|c|c|}
\hline Clinical characteristics & $\begin{array}{l}\text { OM group (\%) } \\
(n=21)\end{array}$ & $\begin{array}{l}\text { NOM group }(\%) \\
(n=467)\end{array}$ & P value ${ }^{c}$ & \\
\hline \multicolumn{5}{|l|}{ Sex ${ }^{a}$} \\
\hline Male & $16(76.2)$ & $401(85.9)$ & \multirow[t]{2}{*}{0.219} & \\
\hline Female & $5(23.8)$ & $66(14.1)$ & & \\
\hline \multicolumn{5}{|l|}{ Age (years) ${ }^{b}$} \\
\hline Mean \pm SD & $57.7 \pm 12.2$ & $51.5 \pm 13.8$ & 0.044 & $\begin{array}{l}\text { Notes: aChi-square } \\
\text { test was used. }\end{array}$ \\
\hline History of HBVa & & & \multirow{3}{*}{0.018} & bStudent's t-test was \\
\hline With (+) & $18(85.7)$ & $267(57.2)$ & & used. cComparison \\
\hline Without (-) & $3(14.3)$ & $200(42.8)$ & & 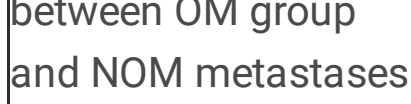 \\
\hline \multicolumn{3}{|l|}{ Tumor clinical stage $\mathrm{a}^{\mathrm{a}}$} & \multirow{5}{*}{0.084} & group. $\mathrm{P}<0.05$ was \\
\hline Stage 2 & $0(0)$ & $15(3.2)$ & & $\begin{array}{l}\text { considered } \\
\text { statistically }\end{array}$ \\
\hline Stage 3 & $3(14.3)$ & $98(21.0)$ & & significant. \\
\hline Stage 4 & $5(23.8)$ & $33(7.1)$ & & Abbreviations: OM, \\
\hline Unknown & $13(61.9)$ & $321(68.7)$ & & ocular metastasis; \\
\hline \multicolumn{4}{|l|}{ Pathological type ${ }^{a}$} & $\begin{array}{l}\text { NOM, non-ocular, } \\
\text { other sites of }\end{array}$ \\
\hline Hepatocellular carcinoma (HCC) & $0(0)$ & $38(8.1)$ & \multirow[t]{4}{*}{0.327} & metastasis; SD, \\
\hline Cholangiocarcinoma & $0(0)$ & $24(5.1)$ & & standard deviation; \\
\hline Mixed hepatocellular carcinoma & $0(0)$ & $2(0.4)$ & & $\begin{array}{l}\text { HBV, hepatitis B virus; } \\
\text { TACE, transcatheter }\end{array}$ \\
\hline Unknown & $21(0)$ & $403(86.4)$ & & arterial \\
\hline \multicolumn{4}{|l|}{ Treatment $^{\mathrm{a}}$} & chemoembolization \\
\hline Surgery & $4(19.0)$ & $85(18.2)$ & \multirow[t]{7}{*}{0.261} & \multirow{7}{*}{$\begin{array}{l}\text { Table } 2 \text { The serum } \\
\text { indicators of patients } \\
\text { with metastases of } \\
\text { primary liver cancer }\end{array}$} \\
\hline Chemotherapy & $3(14.3)$ & $38(8.1)$ & & \\
\hline Protect liver treatment & $9(42.8)$ & $137(29.3)$ & & \\
\hline TACE & $3(14.3)$ & $148(31.6)$ & & \\
\hline Radiation and chemotherapy & $1(4.8)$ & $7(1.5)$ & & \\
\hline Refuse treatment & $1(4.8)$ & $32(6.8)$ & & \\
\hline Other & $0(0)$ & $21(4.5)$ & & \\
\hline
\end{tabular}




\begin{tabular}{|lllll|}
\hline Serum indicators & OM group & NOM group & $\mathbf{t}$ & P value \\
Tumor markers & & & & \\
AFP $(\mathrm{ng} / \mathrm{ml})$ & $1048.80 \pm 273.95$ & $559.00 \pm 553.48$ & 7.529 & $<0.001$ \\
CEA $(\mathrm{ng} / \mathrm{ml})$ & $19.78 \pm 46.90$ & $19.93 \pm 82.97$ & -0.008 & 0.994 \\
CA-125 $(\mathrm{U} / \mathrm{ml})$ & $481.74 \pm 356.55$ & $167.26 \pm 318.36$ & 4.404 & $<0.001$ \\
CA-199 $(\mathrm{U} / \mathrm{ml})$ & $221.34 \pm 338.41$ & $156.12 \pm 292.84$ & 0.992 & 0.322 \\
CA-153 (u/ml) & $18.71 \pm 12.76$ & $22.36 \pm 25.41$ & -0.655 & 0.513 \\
CA-724 (U/ml) & $8.60 \pm 9.76$ & $6.86 \pm 7.16$ & 1.070 & 0.285 \\
Blood lipid indicators & & & & \\
TC $(\mathrm{mmol} / \mathrm{L})$ & $4.63 \pm 1.87$ & $4.12 \pm 1.46$ & 1.566 & 0.118 \\
TG $(\mathrm{mmol} / \mathrm{L})$ & $1.82 \pm 1.38$ & $1.24 \pm 0.88$ & 1.895 & 0.072 \\
HDL $(\mathrm{mmol} / \mathrm{L})$ & $1.57 \pm 1.30$ & $1.37 \pm 0.95$ & 0.905 & 0.366 \\
LDL $(\mathrm{mmol} / \mathrm{L})$ & $2.91 \pm 1.97$ & $2.40 \pm 1.27$ & 1.148 & 0.265 \\
ApoA1 $(\mathrm{g} / \mathrm{L})$ & $1.64 \pm 0.47$ & $1.55 \pm 0.47$ & 0.825 & 0.410 \\
ApoB $(\mathrm{g} / \mathrm{L})$ & $1.01 \pm 0.59$ & $0.97 \pm 0.58$ & 0.299 & 0.765 \\
Lp $(\mathrm{a})(\mathrm{mg} / \mathrm{L})$ & $148.20 \pm 182.73$ & $224.15 \pm 238.04$ & -1.407 & 0.16 \\
Calcium $(\mathrm{mmol} / \mathrm{L})$ & $2.12 \pm 0.24$ & $2.16 \pm 0.28$ & -0.665 & 0.506 \\
Hb (g/L) & $114.71 \pm 36.00$ & $116.02 \pm 24.02$ & -0.237 & 0.812 \\
Ferritin $(\mu \mathrm{g} / \mathrm{L})$ & $235.13 \pm 243.46$ & $264.73 \pm 207.33$ & -0.635 & 0.526 \\
ALP $(\mathrm{U} / \mathrm{L})$ & $225.95 \pm 122.46$ & $199.78 \pm 189.18$ & 0.628 & 0.530 \\
\hline
\end{tabular}

Notes: Student's $t$-test was uesd. $P<0.05$ represented statistically significant.

Abbreviations: $\mathrm{OM}$, ocular metastasis; NOM, non-ocular, other sites of metastasis; TC, total cholesterol; TG, triglycerides; HDL, high-density lipoprotein; LDL, low-density lipoprotein; ApoA1, apolipoprotein $A 1$; ApoB, apolipoprotein B; Lp(a), lipoprotein a; Hb, hemoglobin; ALP, alkaline phosphatase.

Table 3 Independent risk factors of OM in patients with primary liver cancer

\begin{tabular}{|lllll|}
\hline Factor & $\mathrm{B}$ & $\operatorname{Exp}(\mathrm{B})$ & $\mathrm{OR}(95 \% \mathrm{Cl})$ & $\mathrm{P}$ \\
AFP & 0.001 & 1.001 & $1.001-1.002$ & $<0.001$ \\
CA-125 & 0.001 & 1.001 & $1.001-1.002$ & $<0.001$ \\
\hline
\end{tabular}

Notes: Binary logistic analysis was used. $\mathrm{P}<0.05$ represented statistically significant. 
Abbreviations: $\mathrm{B}$, coefficient of regression; $\mathrm{OR}$, odds ratio; $\mathrm{Cl}$ confidence interval; $\mathrm{OM}$ ocular metastasis.

Table 4 The AUC, sensitivity, specificity and cut-off value for single risk factors in predicting OM from primary liver cancer

\begin{tabular}{|llllll|}
\hline Factor & AUC & $\begin{array}{l}\text { Sensitivity } \\
(\%)\end{array}$ & $\begin{array}{l}\text { Specificity } \\
(\%)\end{array}$ & $\begin{array}{l}\text { Cut-off value } \\
\mathrm{U} / \mathrm{ml}\end{array}$ & $\mathrm{P}$ \\
\hline AFP & 0.739 & 95.2 & 56.8 & 957.2 & $<0.001$ \\
CA-125 & 0.810 & 90.2 & 64.0 & 14.25 & $<0.001$ \\
AFP+CA-125 & 0.875 & 76.2 & 88.4 & - & $<0.001$ \\
\hline
\end{tabular}

Notes: Sensitivity and specificity were obtained at the point of cut-off value. $P<0.05$ represented statistically significant.

Abbreviations: AUC, area under the curve; OM, ocular metastasis

\section{Figures}

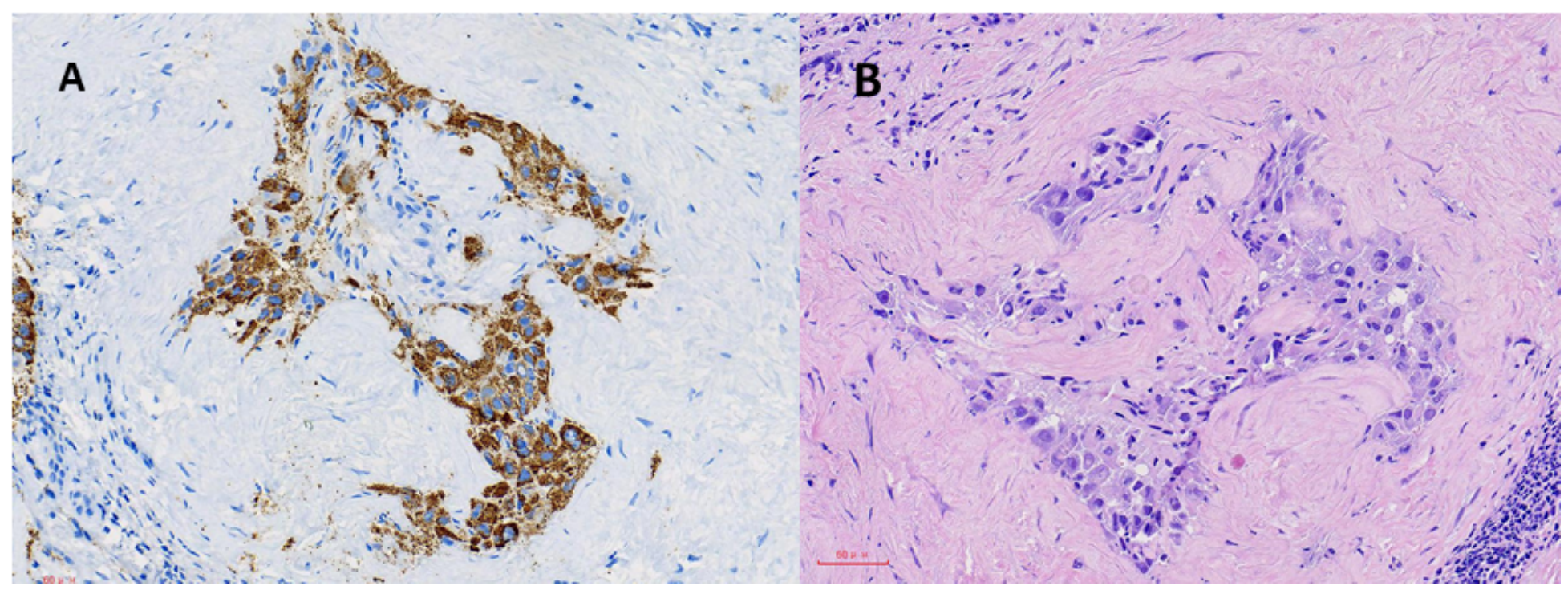

Figure 1

$\mathrm{A} \square \mathrm{HE}$ staining of primary liver cancer tissues. $\mathrm{B} \square \mathrm{IHC}$ staining of primary liver cancer tissues. Notes: $\mathrm{HE}$

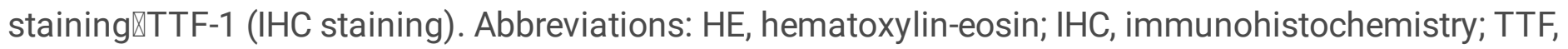
transcription factor; 


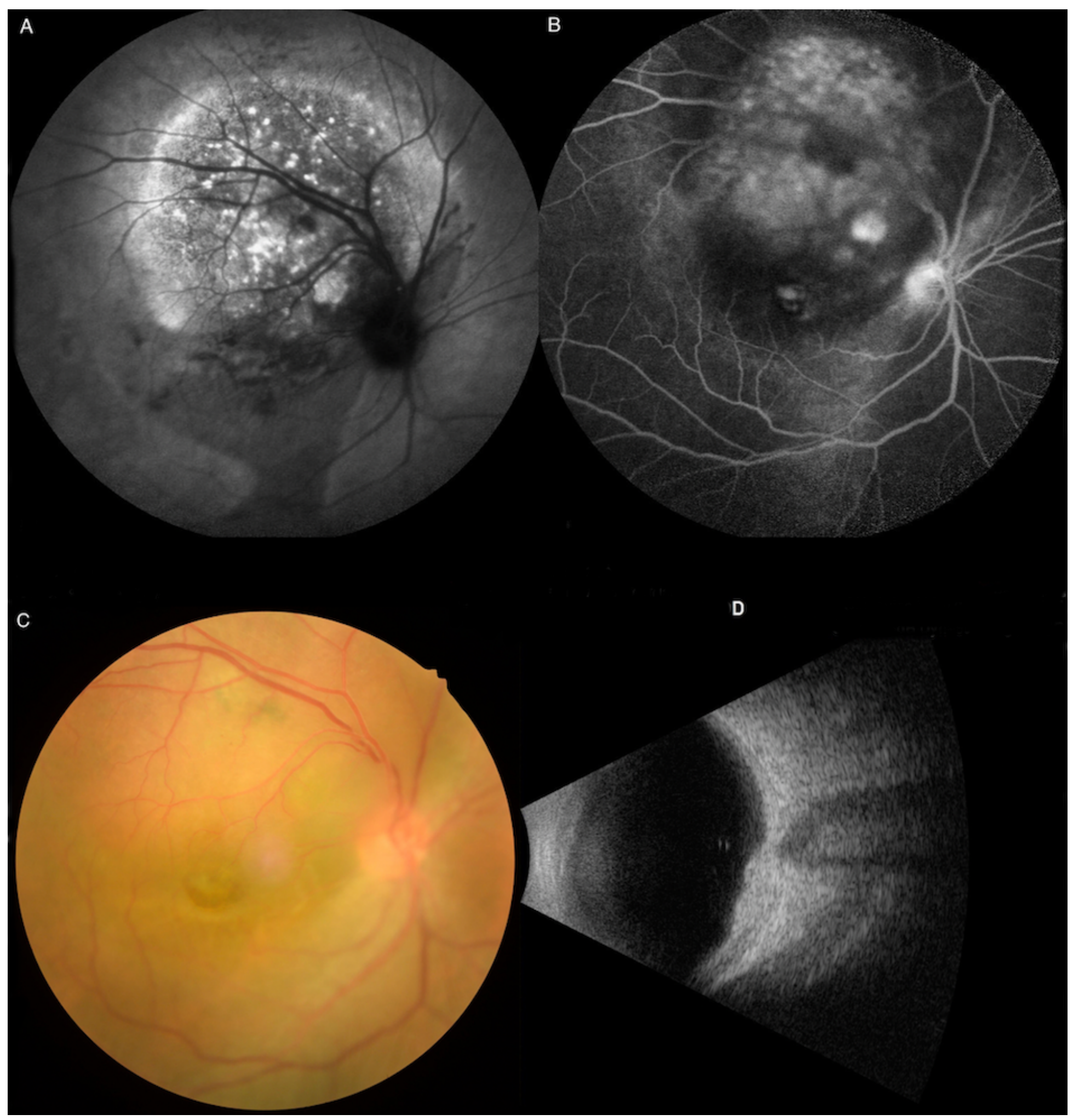

Figure 2

$\mathrm{A} \square \mathrm{B} \otimes$ Fundus fluorescein angiography in patients with primary liver cancer $\mathrm{C} \square \mathrm{D} \otimes$ Funduscopy in patients with primary liver cancer 
A

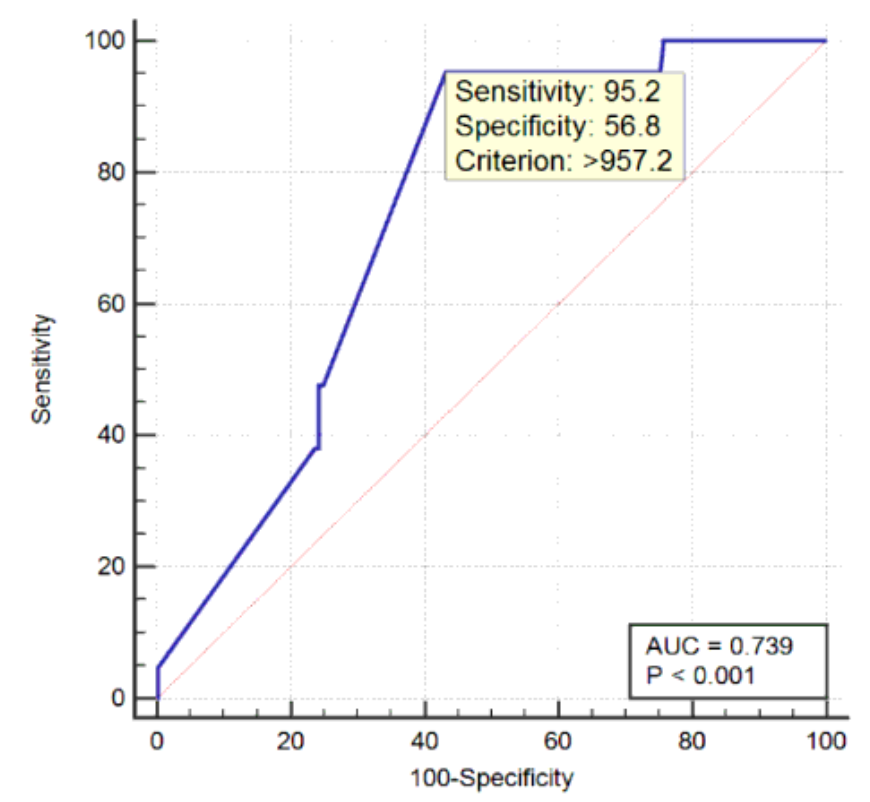

B

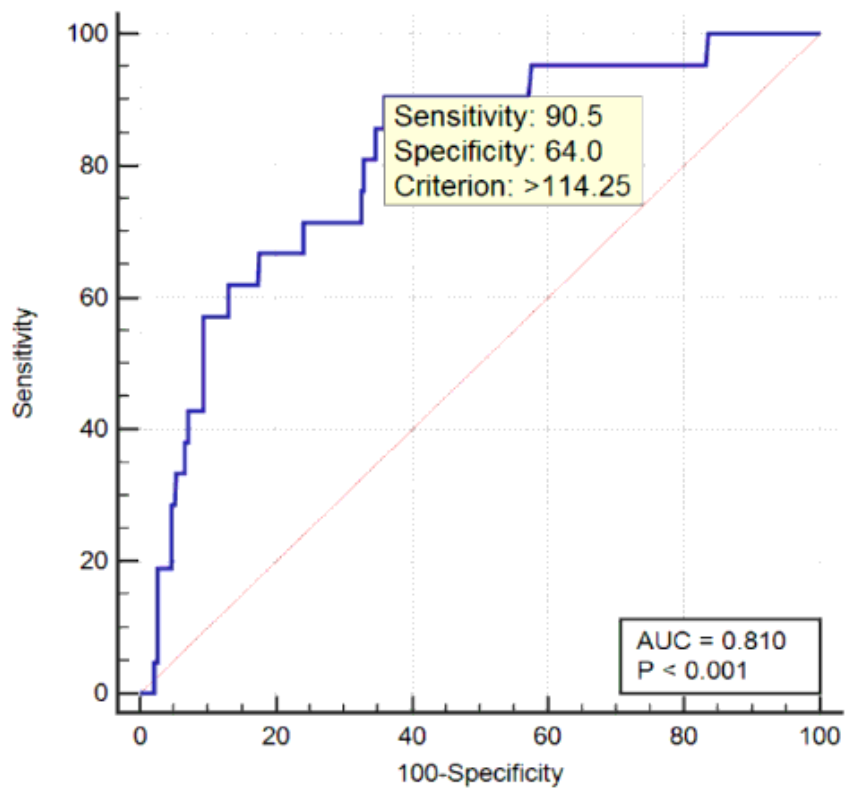

\section{Figure 3}

ROC curve of independent risk factors for ocular metastasis from liver cancer Notes: A. ROC curve of AFP; B. ROC curve of CA-125. Abbreviations: AUC, area under the curve; ROC, receiver operating curve. 


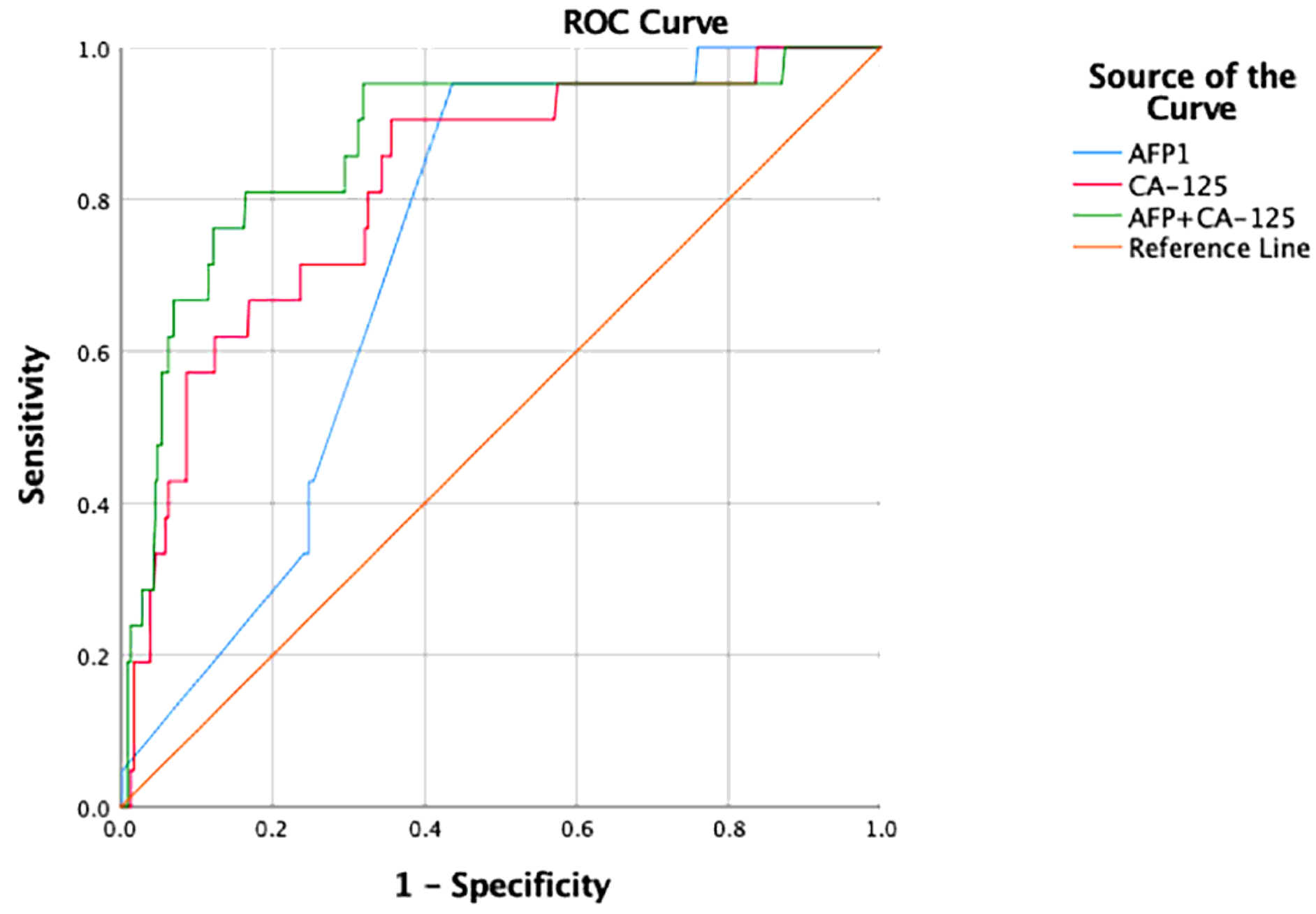

Figure 4

ROC curve of respective and combination of independent risk factors for ocular metastasis from liver cancer Abbreviations: AUC, area under the curve; ROC, receiver operating curve. 